\title{
Structure factor of dilute ring polymers
}

\author{
Pasquale Calabrese \\ Scuola Normale Superiore and INFN - Sezione di Pisa \\ I-56100 Pisa, ITALY \\ Internet: Pasquale.Calabrese@df .unipi.it \\ Andrea Pelissetto \\ Dipartimento di Fisica and INFN - Sezione di Roma I \\ Università degli Studi di Roma "La Sapienza" \\ I-00185 Roma, ITALY \\ Internet: Andrea.Pelissetto@roma1.infn.it \\ Ettore Vicari \\ Dipartimento di Fisica and INFN - Sezione di Pisa \\ Università degli Studi di Pisa \\ I-56100 Pisa, ITALY \\ Internet: Ettore.Vicari@df.unipi.it
}

November 13, 2018

\begin{abstract}
We consider ring polymers in good solvents in the dilute limit. We determine the structure factor and the monomer-monomer distribution function. We compute accurately the asymptotic behavior of these functions for small and large momenta and distances by using field-theoretical methods. Phenomenological expressions with the correct asymptotic behaviors are also given.
\end{abstract}




\section{Introduction}

The statistical properties of dilute polymers in good solvents have been the subject of extensive studies during the years [1 [5]. A significant understanding of the problem was reached when it was realized that long polymers could be modelled by chains with an excluded-volume interaction. This allowed the introduction of simplified theoretical models which could be analyzed more easily. From a theoretical point of view, an important step forward was made by de Gennes [6], who proved that the statistical properties of dilute polymers could be obtained as the limit $n \rightarrow 0$ of the $n$-component $\phi^{4}$ theory, opening the field to the many methods that have been developed for the study of the critical behavior of spin systems.

In nature polymers may have many different geometrical conformations. In this paper we will focus on ring polymers and we will determine the structure factor and the monomer-monomer distribution function. For this purpose we will use the mapping with the $\lambda \phi^{4}$ theory [6] 10] and some recent theoretical results for the twopoint function of biquadratic operators [11, 12. Indeed, we show that the structure factor is directly related to the energy-energy correlation function. Then, by extending the results available for the end-to-end distribution function 13 20], we compute its large- and small-momentum behavior. Moreover, by using an interpolation formula based on a dispersive approach [12, 21], we obtain a general expression valid for all momenta, i.e. for all values of the radiation (neutron) wavelength. We also investigate the monomer-monomer distribution function that is closely related to the structure factor by a Fourier transform. Its properties for small and large distances are investigated in detail.

The paper is organized as follows. In Sec. 2 we define the structure factor and the monomer-monomer distribution function for $N$-step ring polymers. In Sec. 3.1 we show that the computation of the structure factor is equivalent to the calculation of the energy-energy correlation function in the $n$-component $\lambda \phi^{4}$ theory in the limit $n \rightarrow 0$. Field-theoretical results for such a correlation function are reviewed in Sec. 3.2. In Sec. 4 we study the structure factor. In particular, we determine its small- $q^{2}$ behavior extending the classical Guinier formula, its large- $q^{2}$ behavior, and give a general interpolation formula that has the correct asymptotic behavior for $q^{2} \rightarrow 0$ and $q^{2} \rightarrow \infty$. Finally, in Sec. 5 we report the corresponding expressions for the monomer-monomer distribution function. We also verify that the phenomenological expression often used for the end-to-end distribution function for linear polymers 15 18 also provides a good approximation to the monomer-monomer distribution function considered here.

\section{Definitions}

We consider a monodisperse ensemble of ring polymers with $N$ monomers, labelling the monomers from 1 to $N$. Then, we consider two monomers $i$ and $j$ of the walk and the unnormalized distribution $d_{i j, N}(\vec{r})$ of the distance $\vec{r}$ between the two monomers. Such a distribution function depends only on the relative position $|i-j|$ of the 
monomers along the polymer. Then, we define its average over all pairs $i, j$, i.e.

$$
p_{N}(\vec{r})=\frac{1}{N^{2}} \sum_{i, j=1}^{N} d_{i j, N}(\vec{r}),
$$

the corresponding normalized distribution

$$
P_{N}(\vec{r})=\frac{p_{N}(\vec{r})}{\int d^{d} \vec{s} p_{N}(\vec{s})},
$$

and the mean squared radius of gyration

$$
R_{g, N}^{2}=\frac{1}{2} \int d^{d} \vec{s}|s|^{2} P_{N}(\vec{s}) .
$$

On a lattice, $d_{i j, N}(\vec{r})$ can be identified with the number of $N$-step lattice rooted selfavoiding polygons such that monomer $i$ is in the origin and monomer $j$ is in $\vec{r}$. Note that

$$
p_{N} \equiv \sum_{s} d_{i j, N}(\vec{s})=\sum_{s} p_{N}(\vec{s})
$$

is the total number of $N$-step lattice rooted polygons.

The structure factor is defined by

$$
S_{N}(\vec{q})=\int d^{d} \vec{s} e^{i \vec{q} \cdot \vec{s}} P_{N}(\vec{s}),
$$

which, by definition, satisfies $S_{N}(0)=1$. For elastic scattering, the momentum $\vec{q}$ is directly related to the wavelength $\lambda$ of the incoming radiation in the scattering medium and to the scattering angle $\theta$ by

$$
|q|=\frac{4 \pi}{\lambda} \sin \frac{\theta}{2} .
$$

For comparison, we will also consider linear polymers and correspondingly we define the mean squared end-to-end distance $\widehat{R}_{e, N}^{2}$ and the mean squared radius of gyration $\widehat{R}_{g, N}^{2}$. In the limit $N \rightarrow \infty$, the radius of gyration and the end-to-end distance diverge with the same critical exponent $\nu$, i.e.

$$
\begin{aligned}
& R_{g, N}^{2}=a_{g} N^{2 \nu}, \\
& \widehat{R}_{g, N}^{2}=\hat{a}_{g} N^{2 \nu}, \\
& \widehat{R}_{e, N}^{2}=\hat{a}_{e} N^{2 \nu} .
\end{aligned}
$$

In two dimensions the universal exponent $\nu$ is given by $\nu=3 / 4$, while in three dimensions

$$
\nu= \begin{cases}0.5877(6) & \text { Ref. [22] } \\ 0.58758(7) & \text { Ref. [23]. }\end{cases}
$$

See Ref. [24] for other numerical and theoretical estimates. 
In the limit $N \rightarrow \infty$, the normalized distribution $P_{N}(\vec{r})$ and the structure factor $S_{N}(\vec{q})$ obey general scaling laws. More precisely, for $N \rightarrow \infty,|r| \rightarrow \infty,|q| \rightarrow 0$, with $\vec{\rho} \equiv \vec{r} / R_{g, N}$ and $\vec{Q} \equiv q R_{g, N}$ fixed, we have

$$
\begin{aligned}
& P_{N}(\vec{r}) \approx \frac{1}{R_{g, N}^{d}} f(\rho), \\
& S_{N}(\vec{q}) \approx s(Q) .
\end{aligned}
$$

These functions satisfy the following normalization conditions:

$$
\begin{aligned}
\int d \vec{\rho} f(\rho) & =1 & \int d \vec{\rho} \rho^{2} f(\rho) & =2, \\
s(0) & =1 & \left.\frac{d s(Q)}{d Q^{2}}\right|_{Q=0} & =-\frac{1}{3} .
\end{aligned}
$$

\section{$3 \quad$ Field-theoretical results}

\subsection{Generalities}

We wish now to derive some general properties of the functions we have introduced above. We will use the Laplace-de Gennes transform method [3, 66, 18] and the results for biquadratic correlation functions in $n$-vector models obtained in Refs. 111, 12.

For this purpose, consider a hypercubic lattice in $d$ dimensions and the $n$-vector model Hamiltonian

$$
H=-\beta \sum_{\langle r s\rangle} \boldsymbol{\sigma}_{r} \cdot \boldsymbol{\sigma}_{s},
$$

where $\boldsymbol{\sigma}_{r}$ is a unit $n$-dimensional vector and the sum is extended over all lattice nearest-neighbor sites $\langle r s\rangle$. Then, consider the correlation function

$$
C_{\mu}(\vec{r} ; \beta) \equiv\left\langle\left(\boldsymbol{\sigma}_{0} \cdot \boldsymbol{\sigma}_{\mu}\right)\left(\boldsymbol{\sigma}_{r} \cdot \boldsymbol{\sigma}_{r+\mu}\right)\right\rangle-\left\langle\left(\boldsymbol{\sigma}_{0} \cdot \boldsymbol{\sigma}_{\mu}\right)\right\rangle^{2},
$$

where $\vec{\mu}$ is an arbitrary lattice unit vector. By using standard results (see, e.g., Refs. [2, [3]), it is easy to verify that

$$
\lim _{n \rightarrow 0}\left[\frac{1}{n} C_{\mu}(\vec{r} ; \beta)\right]=\sum_{N} \beta^{N-2} c_{N, \mu}(\vec{r}),
$$

where $c_{N, \mu}(\vec{r})$ is the number of $N$-step lattice self-avoiding polygons that go through the lattice links $\langle 0, \vec{\mu}\rangle$ and $\langle\vec{r}, \vec{r}+\vec{\mu}\rangle$. In the limit $N \rightarrow \infty,|r| \rightarrow \infty$ we are interested in, $c_{N, \mu}(\vec{r})$ is proportional to the number of lattice polygons $p_{N}(\vec{r})$ going through 0 and $\vec{r}$. Then

$$
\lim _{n \rightarrow 0}\left[\frac{1}{n} C_{\mu}(\vec{r} ; \beta)\right] \approx \text { const } \times \sum_{N} \beta^{N} p_{N} P_{N}(\vec{r}) .
$$

Now, let us consider the left-hand side. In the critical limit it can be identified with the energy-energy correlation function in the $\lambda \phi^{4}$ theory. More precisely, if $t_{\sigma} \equiv\left(\beta_{c}-\beta\right) / \beta_{c} \rightarrow 0$ is the reduced temperature in the $n$-vector model, we have

$$
C_{\mu}(\vec{r} ; \beta) \approx \text { const } \times G_{E}\left(\vec{r}, k t_{\sigma}\right),
$$


where $k$ is a constant and

$$
G_{E}(\vec{r}, t) \equiv\left\langle\phi^{2}(0) \phi^{2}(\vec{r})\right\rangle_{t}-\left\langle\phi^{2}(0)\right\rangle_{t}^{2}
$$

where $\langle\cdot\rangle_{t}$ indicates that the average should be taken at reduced temperature $t$ in the $\lambda \phi^{4}$ theory.

For $N \rightarrow \infty$ one can then derive (see, e.g., Ref. [3])

$$
P_{N}(\vec{r}) \approx \text { const } \times \int_{-i \infty}^{+i \infty} \frac{d t}{2 \pi i} e^{N t} \lim _{n \rightarrow 0}\left[\frac{1}{n} G_{E}(\vec{r}, k t)\right]
$$

where the integral is over a line parallel to the imaginary axis that leaves all singularities in the left-hand side.

\subsection{Behavior of $G_{E}(\vec{r}, t)$}

\subsubsection{General results}

The general properties of $G_{E}(\vec{r}, t)$ and of its Fourier transform $\widetilde{G}_{E}(\vec{q}, t)$ were studied in Refs. [11, 12]. We will review them here. In the scaling limit $t \rightarrow 0^{+}$, we have

$$
\widetilde{G}_{E}(\vec{q}, t)=A_{E}^{+} t^{-\alpha} f_{E}(|q| \xi)\left[1+O\left(t^{\alpha}, t^{\Delta}\right)\right]
$$

where $\alpha=2-d \nu(\alpha=0.23726(21)$ in three dimensions $), f_{E}(y)$ is a universal function, and $\xi$ is the second-moment correlation length defined in terms of the two-point function of the fundamental field $\widetilde{G}_{\phi}(\vec{q}, t)$,

$$
\xi^{2}=-\left.\widetilde{G}_{\phi}(0, t)^{-1} \frac{\partial \widetilde{G}_{\phi}(\vec{q}, t)}{\partial q^{2}}\right|_{q^{2}=0}
$$

Notice the presence of two types of corrections in Eq. (3.8). First of all, there are the corrections due to the leading irrelevant operator with exponent $\Delta$. Additionally, there are corrections with exponent $\alpha$ due to the presence of a background term. Note that both in two and three dimensions [25] $\alpha<\Delta$, and thus the leading correction is due to the background term.

The function $f_{E}(y)$ has a regular expansion for $y \rightarrow 0$,

$$
f_{E}(y)=1+\sum_{n=1}^{\infty} e_{n} y^{2 n}
$$

while for $y \rightarrow \infty$ it has the nonanalytic behavior

$$
f_{E}(y) \approx E_{1} y^{-\alpha / \nu}\left[1+E_{2} y^{-(1-\alpha) / \nu}+E_{3} y^{-1 / \nu}\right]
$$

For generic values of $y$, the universal function $f_{E}(y)$ can be written in the form

$$
f_{E}(y)=1-\frac{y^{2} E_{1}}{\pi} \sin \left(\frac{\pi \alpha}{2 \nu}\right) \int_{4 S_{M}^{+}}^{\infty} d x \frac{x^{-1-\alpha /(2 \nu)}}{x+y^{2}} F_{E}(x),
$$


where $E_{1}$ is the coefficient appearing in Eq. (3.11), $S_{M}^{+}$is a constant, and $F_{E}(y)$ is the spectral function. The constant $S_{M}^{+}$is universal and it is defined by $S_{M}^{+} \equiv \xi^{2} / \xi_{\text {gap }}^{2}$, where $\xi_{\text {gap }}$ is the exponential correlation length that determines the large-distance behavior of the two-point function $G_{\phi}(\vec{r}, t)$ of the field $\phi$. The constant $E_{1}$ and the spectral function are related by the sum rule

$$
\frac{E_{1}}{\pi} \sin \left(\frac{\pi \alpha}{2 \nu}\right) \int_{4 S_{M}^{+}}^{\infty} d x x^{-1-\alpha /(2 \nu)} F_{E}(x)=1
$$

The representation (3.12) is exact under rather mild assumptions [12, 21]. Approximate expressions can be obtained by choosing approximate forms for the spectral function. As in Ref. [12, we choose

$$
F_{E}(x)=1+E_{2} \Phi_{2} x^{-(1-\alpha) /(2 \nu)}+E_{3} \Phi_{3} x^{-1 /(2 \nu)},
$$

where $E_{2}$ and $E_{3}$ are the constants that parametrize the large- $q^{2}$ behavior in Eq. (3.11) and

$$
\begin{aligned}
\Phi_{2} & =\cos \frac{\pi(1-\alpha)}{2 \nu}+\sin \frac{\pi(1-\alpha)}{2 \nu} \cot \frac{\pi \alpha}{2 \nu} \\
\Phi_{3} & =\cos \frac{\pi}{2 \nu}+\sin \frac{\pi}{2 \nu} \cot \frac{\pi \alpha}{2 \nu}
\end{aligned}
$$

We further define

$$
\begin{aligned}
g(p ; x) \equiv-\frac{\pi}{\sin \left(\frac{\pi \alpha}{2 \nu}+\pi p\right)} x^{-1-p-\alpha /(2 \nu)} & \\
& \quad+\frac{2 \nu\left(4 S_{M}^{+}\right)^{-p-\alpha /(2 \nu)}}{(\alpha+2 p \nu)\left(x+4 S_{M}^{+}\right)}{ }_{2} F_{1}\left(1,1 ; 1-p-\frac{\alpha}{2 \nu} ; \frac{4 S_{M}^{+}}{x+4 S_{M}^{+}}\right)
\end{aligned}
$$

where ${ }_{2} F_{1}(a, b ; c ; z)$ is a hypergeometric function [26]. Then

$$
f_{E}(y)=1-\frac{y^{2} E_{1}}{\pi} \sin \left(\frac{\pi \alpha}{2 \nu}\right)\left[g\left(0 ; y^{2}\right)+E_{2} \Phi_{2} g\left(\frac{1-\alpha}{2 \nu} ; y^{2}\right)+E_{3} \Phi_{3} g\left(\frac{1}{2 \nu} ; y^{2}\right)\right]
$$

or, by using the sum rule (3.13),

$$
f_{E}(y)=\frac{E_{1}}{\pi} \sin \left(\frac{\pi \alpha}{2 \nu}\right)\left[g\left(-1 ; y^{2}\right)+E_{2} \Phi_{2} g\left(-1+\frac{1-\alpha}{2 \nu} ; y^{2}\right)+E_{3} \Phi_{3} g\left(-1+\frac{1}{2 \nu} ; y^{2}\right)\right] \text {. }
$$

From Eq. (3.8) we also obtain

$$
G_{E}(\vec{r}, t)=\frac{A_{E}^{+} t^{-\alpha}}{\xi^{d}} \widetilde{f}_{E}(s)\left[1+O\left(t^{\alpha}, t^{\Delta}\right)\right]
$$

where $\vec{s} \equiv \vec{r} / \xi$, and

$$
\widetilde{f}_{E}(s)=\int \frac{d^{d} \vec{y}}{(2 \pi)^{d}} f_{E}(y) e^{i \vec{s} \cdot \vec{y}}
$$


Table 1: Estimates of the small-momentum expansion coefficients by using the fixeddimension expansion in $d=3(d=3)$ and the $\epsilon$ expansion ( $\epsilon$-exp). "final" labels our subjective final estimates. Perturbative series from Ref. [12].

\begin{tabular}{clcccc}
\hline \hline & $e_{1}$ & $e_{2}$ & $e_{3}$ & $e_{4}$ & $e_{5}$ \\
\hline$(d=3)$ & $-0.0322(11)$ & $0.00382(15)$ & $-0.000597(23)$ & $1.06(4) \times 10^{-4}$ & $-2.03(8) \times 10^{-5}$ \\
$(\epsilon$-exp $)$ & $-0.0323(7)$ & $0.00398(11)$ & $-0.000636(10)$ & $1.14(4) \times 10^{-4}$ & $-2.22(3) \times 10^{-5}$ \\
final & $-0.0323(10)$ & $0.00390(15)$ & $-0.000620(30)$ & $1.10(8) \times 10^{-4}$ & $-2.15(15) \times 10^{-5}$ \\
\hline \hline
\end{tabular}

For $s \rightarrow \infty, \tilde{f}_{E}(s)$ decays exponentially as

$$
\tilde{f}_{E}(s)=A s^{p} \exp \left(-\frac{s \xi}{\xi_{E, \text { gap }}}\right),
$$

where

$$
\xi_{E, \text { gap }}=\frac{1}{2} \xi_{\text {gap }}=\frac{\xi}{2 \sqrt{S_{M}^{+}}},
$$

and $p$ is an exponent that can in principle be computed perturbatively.

An approximate expression can be obtained from Eq. (3.18):

$$
\tilde{f}_{E}(s)=\frac{E_{1}}{4 \pi^{2} s} \sin \left(\frac{\pi \alpha}{2 \nu}\right)\left[h(0 ; s)+E_{2} \Phi_{2} h\left(\frac{1-\alpha}{2 \nu} ; s\right)+E_{3} \Phi_{3} h\left(\frac{1}{2 \nu} ; s\right)\right],
$$

where

$$
h(p ; s)=2 s^{2 p-2+\alpha / \nu} \Gamma\left(2-2 p-\alpha / \nu, 2 s \sqrt{S_{M}^{+}}\right),
$$

and $\Gamma(\alpha ; x)$ is an incomplete $\Gamma$-function [26].

\subsubsection{Numerical results}

We have introduced in the preceding section several constants that will be computed here.

First, let us consider the constants $e_{n}$ that parametrize the small-momentum expansion of $f_{E}(y)$. Perturbative series were derived in Ref. 112 for $e_{n}, n=1, \ldots, 5$, in the fixed-dimension expansion in three dimensions (four loops) and in $\epsilon$-expansion (three loops). We resummed the perturbative series by using their large-order behavior and performing a conformal mapping as in Ref. [27]. Mean values ad errors were computed using the algorithm of Ref. [28]. For the expansion in fixed dimension we used for the four-point renormalized coupling 29] $\bar{g}^{*}=1.395(15)$ that includes all available estimates [30 32]. The results are reported in Table 1. Note that the two expansions give estimates that agree within error bars, confirming the correctness of our results within the quoted errors. 
We can also use the results of Ref. [12] to compute the large-momentum expansion coefficients $E_{i}$. In $\epsilon \equiv 4-d$ expansion, they are explicitly given by

$$
\begin{aligned}
& E_{1}=1+\frac{\epsilon}{2}+O\left(\epsilon^{2}\right) \\
& E_{2}=-2+\frac{5 \epsilon}{4}+O\left(\epsilon^{2}\right) \\
& E_{3}=2-\frac{7 \epsilon}{4}+O\left(\epsilon^{2}\right) .
\end{aligned}
$$

In the following we will also be interested in the product $E_{1} E_{2}$ given by

$$
E_{1} E_{2}=-2+\frac{\epsilon}{4}+O\left(\epsilon^{2}\right)
$$

The large size of the coefficients makes it difficult to resum the perturbative series. We report here the results obtained by setting $\epsilon=1$ and as error we quote the last coefficient: $E_{1}=1.5(5), E_{2}=-0.75(1.25), E_{3}=0.25(1.75), E_{1} E_{2}=-1.75(25)$. For the sum $E_{2}+E_{3}$ an additional term is known:

$$
E_{2}+E_{3}=-\frac{1}{2} \epsilon+\left(\frac{27}{64}-\frac{\pi^{2}}{24}\right) \epsilon^{2}+O\left(\epsilon^{3}\right),
$$

so that $E_{2}+E_{3}=-0.49(1)$.

We also need the universal ratio $S_{M}^{+}$. In three dimensions it has been estimated by a variety of field-theoretical and exact-enumeration methods [33], obtaining $S_{M}^{+}-1=$ $-3(1) \times 10^{-4}$. In two dimensions, an exact-enumeration study gives $34 S_{M}^{+}-1=$ $1(2) \times 10^{-4}$.

We wish finally to determine the functions $f_{E}(y)$ and $\tilde{f}_{E}(\rho)$ by using Eqs. (3.18) and (3.23). For this purpose, we must fix $\alpha, \nu, S_{M}^{+}, E_{2}$, and $E_{3}$, while $E_{1}$ is fixed by using the sum rule (3.13). For $\nu$ and $\alpha=2-3 \nu$, we use [23] $\nu=0.58758$, while for $S_{M}^{+}$we use the above-reported result. For $E_{2}$ and $E_{3}$, the only available estimates are those obtained in the $\epsilon$-expansion approach. They have a large error, while, apparently, their sum is more precisely determined. We have thus followed the following strategy. We have fixed $E_{2}+E_{3}=-0.49$ using the $\epsilon$-expansion. Then, we have chosen $E_{2}$ and $E_{3}$ so that for small $y$ the approximation gives $1-0.0323 y^{2}$, in agreement with the results of Table 1. In this way we obtain, $E_{2}=-1.38, E_{3}=0.89$, and $E_{1}=1.60$. These estimates are in good agreement with the $\epsilon$-expansion results, confirming that our approximation is reasonably correct for $y \rightarrow \infty$. Moreover, it also nicely reproduces the small $y$-behavior. Indeed, it gives $e_{2} \approx 0.0040, e_{3} \approx-0.00065$, $e_{4} \approx 1.2 \times 10^{-4}$, which are close to the results of Table 1 .

\section{The structure factor $S_{N}(\vec{q})$}

Using Eqs. (2.5), (3.7), and (3.8) we can determine the structure factor $S_{N}(\vec{q})$ in the scaling limit $|q| \rightarrow 0, N \rightarrow \infty$, with $Q \equiv|q| R_{N, g}$ fixed. Neglecting corrections of 
order $N^{-\alpha}$, see the discussion in Sec. 3.2.1, we can write-from now on, the limit $n \rightarrow 0$ is always understood-

$$
S_{N}(\vec{q})=\Gamma(\alpha) \int_{-i \infty}^{+i \infty} \frac{d t}{2 \pi i} e^{t} t^{-\alpha} f_{E}\left(|q| N^{\nu} x_{0} k^{-\nu} t^{-\nu}\right)
$$

where we have written for the second-moment correlation length $\xi, \xi \approx x_{0} t^{-\nu}$ for $t \rightarrow 0$. The nonuniversal factor can be eliminated by introducing the end-to-end distance for linear polymers. A simple calculation gives [3, 18]

$$
\widehat{R}_{e, N}^{2}=2 d \frac{\Gamma(\gamma)}{\Gamma(\gamma+2 \nu)} x_{0}^{2} k^{-2 \nu} N^{2 \nu},
$$

where $\gamma$ is a universal critical exponent. If we define

$$
\kappa \equiv \frac{1}{2 d} \frac{\Gamma(\gamma+2 \nu)}{\Gamma(\gamma)}
$$

then

$$
S_{N}(\vec{q})=\Gamma(\alpha) \int_{-i \infty}^{+i \infty} \frac{d t}{2 \pi i} e^{t} t^{-\alpha} f_{E}\left(\sqrt{\kappa}|q| \widehat{R}_{e, N} t^{-\nu}\right) .
$$

We can now use the results of the preceding section. First, for $|q| \rightarrow 0$ we obtain

$$
S_{N}(\vec{q})=1+\sum_{n=1}^{\infty} e_{n} \frac{\Gamma(\alpha)}{\Gamma(\alpha+2 n \nu)}\left(\kappa q^{2} \widehat{R}_{e, N}^{2}\right)^{n}
$$

In order to express $S_{N}(\vec{q})$ in the scaling form (2.9), we must express $\widehat{R}_{e, N}^{2}$ in terms of $R_{g, N}^{2}$. Using Eqs. (2.3) and (4.5) we have in $d$ dimensions

$$
R_{g, N}^{2}=-\left.d \frac{d S_{N}(\vec{q})}{d q^{2}}\right|_{q^{2}=0}=-d e_{1} \frac{\Gamma(\alpha)}{\Gamma(\alpha+2 \nu)} \kappa \widehat{R}_{e, N}^{2}
$$

which allows to determine the ratio [35]

$$
H \equiv \frac{R_{g, N}^{2}}{\widehat{R}_{e, N}^{2}}=-d e_{1} \kappa \frac{\Gamma(\alpha)}{\Gamma(\alpha+2 \nu)} .
$$

Setting

$$
\bar{\kappa}=-\frac{1}{d e_{1}} \frac{\Gamma(\alpha+2 \nu)}{\Gamma(\alpha)}
$$

we have

$$
S_{N}(\vec{q})=1+\sum_{n=1}^{\infty} e_{n} \frac{\Gamma(\alpha)}{\Gamma(\alpha+2 n \nu)} \bar{\kappa}^{n} Q^{2 n} .
$$

In three dimension $(d=3)$, using [23 $\nu=0.58758(7), \alpha=0.23726(21)$, and 36 $\gamma=1.1575(6)$, we obtain

$$
\kappa=0.21315(12), \quad \bar{\kappa}=2.40(8) .
$$


Thus

$$
H=0.089(3)
$$

and

$$
\begin{aligned}
S_{N}(\vec{q})=1- & \frac{1}{3} Q^{2}+0.061(5) \times Q^{4}-0.0073(7) \times Q^{6} \\
& +0.00063(10) \times Q^{8}-0.000045(8) \times Q^{10}+O\left(Q^{12}\right) .
\end{aligned}
$$

Note that the coefficients decrease quite rapidly, so that this expansion provides a good approximation up to $Q \approx 2$.

We can compare the result (4.11) with the existing estimates. The amplitude $\hat{a}_{e}$, cf. Eq. (2.7), is quite well determined for self-avoiding walks on the cubic lattice: Ref. [22] quotes $\hat{a}_{e}=1.2167(50)$, while Ref. [37] gives $\hat{a}_{e} \approx 1.225$. The amplitude $a_{g}$, cf. Eq. (2.7), for self-avoiding polygons on the cubic lattice can be obtained from the data reported in Ref. [38]. Fitting their data for $R_{g, N}^{2}$ with $a_{g} N^{2 \nu}+b_{g} N^{2 \nu-\Delta}$ and using the estimates of $\nu$ and $\Delta$ of Ref. [23], we obtain $a_{g} \approx 0.102$. Unfortunately, no error bars are reported in Ref. [38] and thus we cannot quote an error on our result. Moreover, the algorithm of Ref. [38] samples only polygons with trivial knot type, and thus some systematic error can in principle be present. Using these estimates, we obtain $H \approx 0.083$, which is reasonably close to our result (4.11).

It is interesting to compute $e_{1}$ and $\bar{\kappa}$ in two dimensions, by using the available estimates of $H, H=0.14605(7)$ (Ref. [39]) and $H=0.1459(2)$ (Ref. 40]). Since $\nu=3 / 4$ and [41] $\gamma=43 / 32$, we obtain $e_{1}=-0.08447(4), \bar{\kappa}=3.3398(16)$.

For $Q^{2} \rightarrow \infty$, we can use Eqs. (4.1) and (3.11) to obtain

$$
S_{N}(\vec{q}) \approx(\alpha-1) E_{1} E_{2}\left(\kappa q^{2} \widehat{R}_{e, N}^{2}\right)^{-1 /(2 \nu)} \approx(\alpha-1) E_{1} E_{2} \bar{\kappa}^{-1 /(2 \nu)} Q^{-1 / \nu} .
$$

In three dimensions, using Eq. (4.10) and the estimate of $E_{1} E_{2}$ of Sec. 3.2.2, we have

$$
S_{N}(\vec{q}) \approx-0.363(10) \times E_{1} E_{2} Q^{-1 / \nu} \approx 0.63(9) \times Q^{-1 / \nu} .
$$

Finally, we can determine an approximate form for $S_{N}(\vec{q})$, by using the interpolation formulae (3.17), (3.18) derived in Sec. 3.2.1. We obtain

$$
\begin{aligned}
S_{N}(\vec{q})= & 1+(\alpha-1) E_{1} E_{2} \bar{\kappa}^{-1 / 2 \nu} Q^{-1 / \nu} \\
& -\frac{E_{1}}{\pi} \sin \left(\frac{\pi \alpha}{2 \nu}\right)\left[\hat{g}\left(0 ; Q^{2}\right)+E_{2} \Phi_{2} \hat{g}\left(\frac{1-\alpha}{2 \nu} ; Q^{2}\right)+E_{3} \Phi_{3} \hat{g}\left(\frac{1}{2 \nu} ; Q^{2}\right)\right],
\end{aligned}
$$

where

$$
\begin{aligned}
& \hat{g}\left(p ; Q^{2}\right)=\frac{2 \nu\left(4 S_{M}^{+}\right)^{-p-\alpha /(2 \nu)}}{\alpha+2 p \nu} \Gamma(\alpha) \int_{-i \infty}^{+i \infty} \frac{d t}{2 \pi i} e^{t} \frac{\bar{\kappa} Q^{2} t^{-\alpha-2 \nu}}{4 S_{M}^{+}+\bar{\kappa} Q^{2} t^{-2 \nu}} \times \\
& \times_{2} F_{1}\left(1,1 ; 1-p-\frac{\alpha}{2 \nu} ; \frac{4 S_{M}^{+}}{4 S_{M}^{+}+\bar{\kappa} Q^{2} t^{-2 \nu}}\right) .
\end{aligned}
$$

The evaluation of $S_{N}(\vec{q})$ requires the evaluation of the integral (4.16). Particular care should be given to the branch cuts. There are indeed three cuts in the complex 


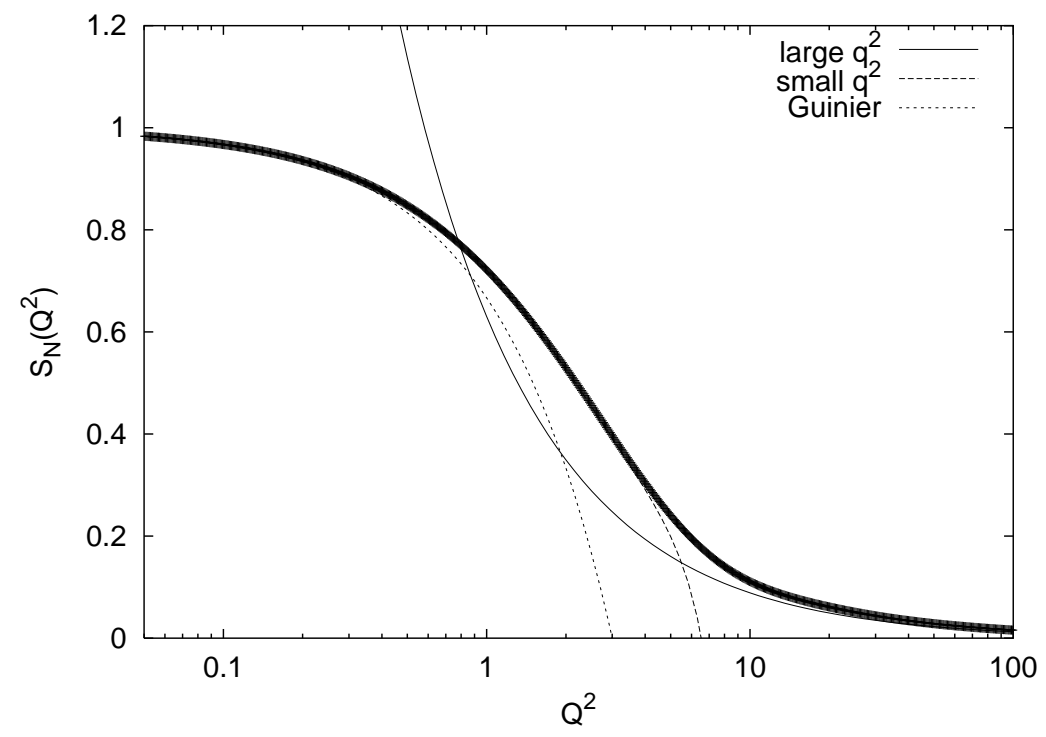

Figure 1: Structure factor $S_{N}(\vec{q})$ versus $Q^{2} \equiv q^{2} R_{g}^{2}$. We also report the large- and small- $Q^{2}$ behavior, cf. Eqs. (4.13) and (4.12), and the classical Guinier formula $S_{N}(\vec{q})=1-Q^{2} / 3$.

$t$-plane: the negative real axis and the lines $t=a \exp [ \pm i \pi /(2 \nu)]$, where $a$ is real satisfying $a \geq\left[\bar{\kappa} Q^{2} /\left(4 S_{M}^{+}\right)\right]^{1 /(2 \nu)}$. In practice, we have found convenient to integrate over the lines $t=a \exp [ \pm i \pi /(2 \nu)]+b$, where $a$ runs over the positive real axis and $b$ is a fixed positive constant that we have taken equal to one.

Using the values of the parameters determined in Sec. 3.2.2, we obtain the curve reported in Fig. 1. We also report the small- $Q^{2}$ expansion (4.12) and the large$Q^{2}$ expansion (4.13). Note that the small- $Q^{2}$ approximation is indistinguishible in the graph from the full curve up to $Q^{2} \approx 5$, while the large- $Q^{2}$ approximation is reasonably accurate for $Q^{2} \gtrsim 40$. Numerical estimates for intermediate values are reported in Table 2 .

We can compare our field-theoretical determination with the numerical results of Ref. [38]. In Fig. 2 we plot $S_{N}(\vec{q})$ in such a way that it can be directly compared with their Fig. 14. We observe a nice quantitative agreement.

\section{Monomer-monomer distribution function $P_{N}(\vec{r})$}

We wish now to compute the distribution function $P_{N}(\vec{r})$, or, more precisely, the scaling function $f(\rho)$ defined in Eq. (2.9). For this purpose we can either use Eq. (2.5) and the results of Sec. 1 for $S_{N}(\vec{q})$, or use directly the field-theoretical results 
Table 2: Estimates of the structor factor $S_{N}(\vec{q})$ for $4 \leq Q^{2} \leq 40$, obtained by using the interpolation formula (4.15).

\begin{tabular}{llll}
\hline \hline$Q^{2}$ & $S_{N}(\vec{q})$ & $Q^{2}$ & $S_{N}(\vec{q})$ \\
\hline 4 & 0.305 & 16 & 0.0733 \\
5 & 0.241 & 18 & 0.0667 \\
6 & 0.196 & 20 & 0.0612 \\
7 & 0.164 & 24 & 0.0527 \\
8 & 0.141 & 28 & 0.0464 \\
9 & 0.124 & 30 & 0.0437 \\
10 & 0.111 & 34 & 0.0393 \\
12 & 0.093 & 38 & 0.0358 \\
14 & 0.082 & 40 & 0.0343 \\
\hline \hline
\end{tabular}

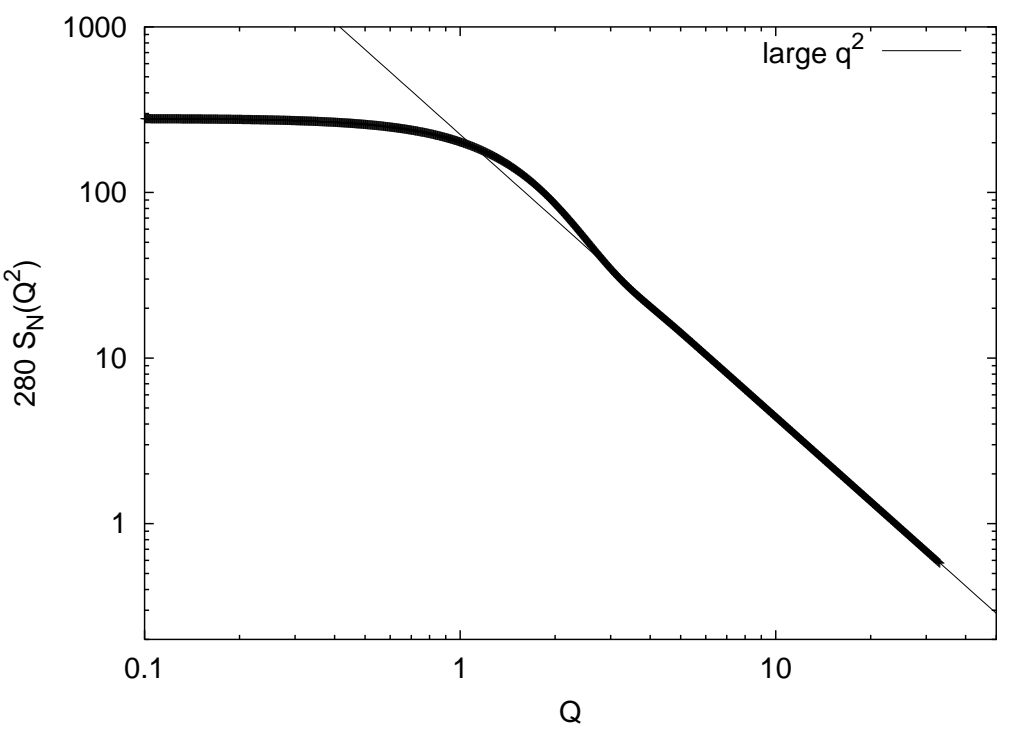

Figure 2: Structure factor $S_{N}(\vec{q})$ versus $Q \equiv|q| R_{g}$. 
available for the scaling function $\widetilde{f}_{E}(\rho)$, cf. Eq. (3.20), and

$$
f(\rho)=\Gamma(\alpha) \bar{\kappa}^{-d / 2} \int_{-i \infty}^{i \infty} \frac{d t}{2 \pi i} e^{t} t^{-\alpha+\nu d} \widetilde{f}_{E}\left(\rho t^{\nu} / \bar{\kappa}^{1 / 2}\right) .
$$

Using the small- $Q^{2}$ result (4.9), we can compute the moments $M_{k}$ of $f(\rho)$, i.e.

$$
M_{k}=\int d^{d} \vec{\rho}|\rho|^{2 k} f(\rho) .
$$

A simple computation gives

$$
M_{k}=(-2 \bar{\kappa})^{k} k ! e_{k} \frac{\Gamma(\alpha)}{\Gamma(\alpha+2 k \nu)} \prod_{n=0}^{k-1}(d+2 n) .
$$

Numerically, in three dimensions, we have $M_{1}=2, M_{2}=7.3(5), M_{3}=37(4), M_{4}=$ $230(35), M_{5}=1780(330)$.

We can use the large- $q^{2}$ expansion to compute the small- $\rho$ behavior of $f(\rho)$. Explicitly, by using Eq. (4.13), we obtain

$$
f(\rho) \approx p_{0} \rho^{1 / \nu-d},
$$

where

$$
p_{0}=(\alpha-1) E_{1} E_{2}(4 \bar{\kappa})^{-1 /(2 \nu)} \pi^{-d / 2} \frac{\Gamma(d / 2-1 /(2 \nu))}{\Gamma(1 /(2 \nu))} .
$$

Numerically in three dimensions, $p_{0}=-0.0250(7) \times E_{1} E_{2}$. If we use the estimate $E_{1} E_{2}=-1.75(25)$ reported in Sec. 3.2.2 we have $p_{0}=0.044(6)$.

Finally, we determine the large- $\rho$ behavior of $f(\rho)$. Starting from Eq. (5.1) and using Eq. (3.21), we have

$$
f(\rho) \approx \Gamma(\alpha) A \bar{\kappa}^{-d / 2-p / 2} \rho^{p} \int_{-i \infty}^{+i \infty} \frac{d t}{2 \pi i} e^{t} t^{-\alpha+\nu d+\nu p} \exp \left(-2 \rho t^{\nu} \sqrt{S_{M}^{+} / \bar{\kappa}}\right) .
$$

The calculation is analogous to that presented in Refs. [14, 18]. We obtain that $f(\rho)$ obeys the Fisher law

$$
f(\rho) \sim \rho^{q} \exp \left(-D \rho^{\delta}\right),
$$

where

$$
\begin{aligned}
\delta & =\frac{1}{1-\nu}, \\
D & =\frac{1-\nu}{\nu}\left(\frac{4 \nu^{2} S_{M}^{+}}{\bar{\kappa}}\right)^{\delta / 2},
\end{aligned}
$$

and $q$ is an exponent that can be expressed in terms of $p$, cf. Eq. (3.21). Numerically, in three dimensions, $\delta=2.425(4), D=0.360(14)$, while in two dimensions $\delta=4$, $D=0.15132(16)$. 


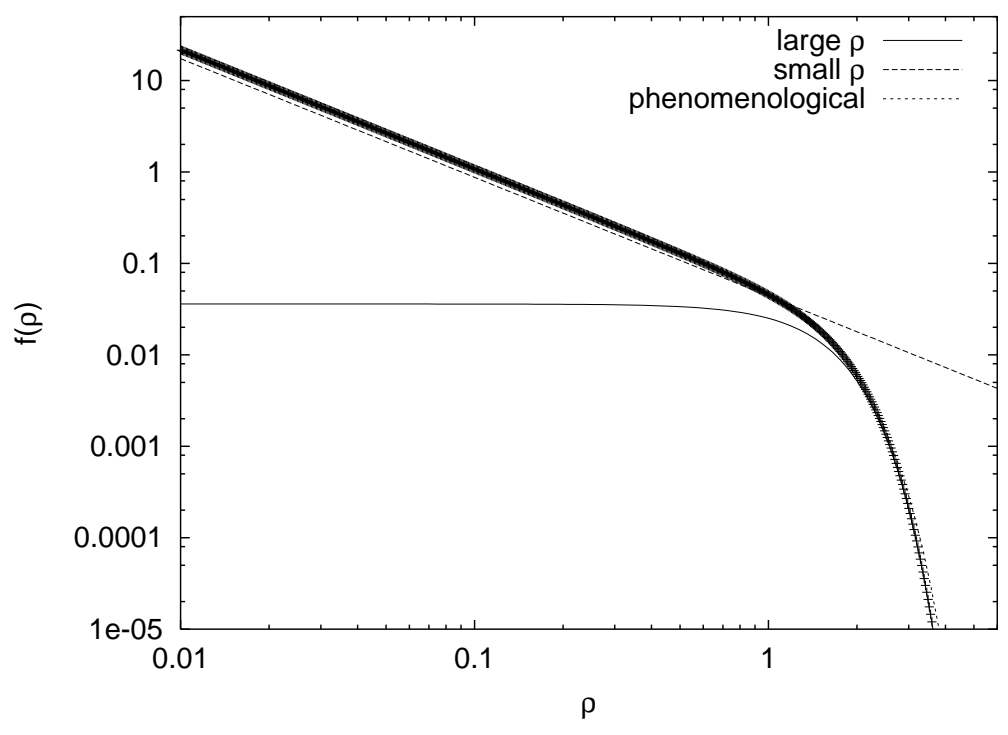

Figure 3: Monomer-monomer scaling function $f(\rho)$ versus $\rho$. We also report the small$\rho$ behavior (5.4), the curve $0.036 e^{-D \rho^{\delta}}$ (labelled "large $\rho$ ") that has the correct asymptotic behavior for $\rho \rightarrow \infty$, and the phenomelogical representation $0.060 \rho^{1 / \nu-3} e^{-0.273 \rho^{\delta}}$.

Finally, we can use the interpolation formula (3.23) to obtain $f(\rho)$ for generic values of $\rho$. We write

$$
f(\rho)=\frac{E_{1}}{4 \pi^{2} \rho} \sin \left(\frac{\pi \alpha}{2 \nu}\right)\left[\hat{h}(0 ; \rho)+E_{2} \Phi_{2} \hat{h}\left(\frac{1-\alpha}{2 \nu} ; \rho\right)+E_{3} \Phi_{3} \hat{h}\left(\frac{1}{2 \nu} ; \rho\right)\right]
$$

where

$$
\hat{h}(p ; \rho)=\frac{2 \Gamma(\alpha)}{\bar{\kappa}}\left(\rho^{2} \bar{\kappa}^{-1}\right)^{p-1+\alpha /(2 \nu)} \int_{-i \infty}^{+i \infty} \frac{d t}{2 \pi i} e^{t} t^{2 p \nu} \Gamma\left(2-2 p-\alpha / \nu, 2 \rho t^{\nu} \sqrt{S_{M}^{+} / \bar{\kappa}}\right) .
$$

Using the parameter values determined in Sec. 3.2.2 we obtain the curve reported in Fig. 3.

In three dimensions the end-to-end distribution function for linear polymers is well described by a very simple phenomenological expression [15] 19],

$$
f_{\mathrm{ph}}(\rho)=A_{\mathrm{ph}} \rho^{\theta} e^{-D_{\mathrm{ph}} \rho^{\delta}},
$$

where $\delta$ and $\theta$ are fixed by the large- $\rho$ and small- $\rho$ behavior, and the constants $A_{\mathrm{ph}}$ and $D_{\mathrm{ph}}$ by the normalization conditions (2.10):

$$
\begin{aligned}
D_{\mathrm{ph}} & =\left[\frac{\Gamma[(1-\nu)(\theta+5)]}{2 \Gamma[(1-\nu)(\theta+3)]}\right]^{\delta / 2}, \\
A_{\mathrm{ph}} & =\frac{\delta}{4 \pi \Gamma[(1-\nu)(\theta+3)]} D_{\mathrm{ph}}^{(1-\nu)(\theta+3)} .
\end{aligned}
$$


An equally good agreement is observed for the function $f(\rho)$ we consider here, at least in the relevant region of $\rho$ not too large $(\rho \lesssim 3)$, if we use $\theta=1 / \nu-3$, and $D_{\mathrm{ph}}=0.273, A_{\mathrm{ph}}=0.060$, see Fig. 通.

\section{References}

[1] P. J. Flory, Principles of Polymer Chemistry (Cornell University Press, Ithaca, NY, 1953).

[2] P. G. de Gennes, Scaling Concepts in Polymer Physics (Cornell University Press, Ithaca, NY, 1979).

[3] J. des Cloizeaux and G. Jannink, Les Polymères en Solution (Les Editions de Physique, Les Ulis, 1987); English translation: Polymers in Solution: Their Modeling and Structure (Oxford University Press, Oxford-New York, 1990).

[4] K. F. Freed, Renormalization-Group Theory of Macromolecules (John Wiley, New York, 1987).

[5] Y. Oono, in Advances in Chemical Physics, Vol. LXI, edited by I. Prigogine and S. A. Rice, p. 301 (1985).

[6] P. G. de Gennes, Phys. Lett. A 38, 339 (1972).

[7] M. Daoud, J. P. Cotton, B. Farnoux, G. Jannink, G. Sarma, H. Benoit, R. Duplessix, C. Picot, and P. G. de Gennes, Macromolecules 8, 804 (1975).

[8] J. des Cloizeaux, J. Phys. (France) 36, 281 (1975).

[9] V. J. Emery, Phys. Rev. B 11, 239 (1975).

[10] C. Aragão de Carvalho, S. Caracciolo, and J. Fröhlich, Nucl. Phys. B 215, 209 (1983).

[11] R. R. Netz and A. Aharony, Phys. Rev. E 55, 2267 (1997).

[12] P. Calabrese, A. Pelissetto, and E. Vicari, "Critical structure factors of bilinear fields in $\mathrm{O}(N)$-vector models," cond-mat/0111160.

[13] M. E. Fisher and B. J. Hiley, J. Chem. Phys. 34, 1253 (1961).

[14] M. E. Fisher, J. Chem. Phys. 44, 616 (1966).

[15] J. Mazur, J. Res. Natl. Bur. Stand. A 69, 355 (1965); J. Chem. Phys. 43, 4354 (1965).

[16] D. S. McKenzie and M. A. Moore, J. Phys. A 4, L82 (1971).

[17] D. S. McKenzie, Phys. Reports 27, 35 (1976). 
[18] J. des Cloizeaux, Phys. Rev. A 10, 1665 (1974); J. Phys. (France) 41, 223 (1980).

[19] S. Caracciolo, M. S. Causo, and A. Pelissetto, J. Chem. Phys. 112, 7693 (2000).

[20] The different proposals appearing in the literature and many old numerical results have been reviewed by Bishop and Clarke [42]. A list of more recent numerical results appears in Ref. [19].

[21] R. A. Ferrell and D. J. Scalapino, Phys. Rev. Lett. 34, 200 (1975).

A. J. Bray, Phys. Rev. B 14, 1248 (1976).

[22] B. Li, N. Madras, and A. D. Sokal, J. Stat. Phys. 80, 661 (1995).

[23] P. Belohorec and B. G. Nickel, "Accurate universal and two-parameter model results from a Monte-Carlo renormalization group study," University of Guelph report (September 1997), unpublished.

[24] A. Pelissetto and E. Vicari, "Critical Phenomena and Renormalization-Group Theory," e-print cond-mat/0012164.

[25] In three dimensions the most precise Monte Carlo estimate of $\Delta$ is $\Delta=0.517 \pm$ $0.007+0.010$ [23]. Perturbative field theory gives a lower estimate [30]: $\Delta=$ $0.478 \pm 0.010(d=3$ expansion) and $\Delta=0.486 \pm 0.016$ ( $\epsilon$-expansion). In two dimensions, the value of $\Delta$ is still the object of intense debate [43 48], some models showing $\Delta=1$, others $\Delta=11 / 16$. These estimates should be compared with [23] $\alpha=0.23726(21)$ in three dimensions and $\alpha=1 / 2$ in two dimensions. Thus, in all cases $\alpha<\Delta$.

[26] I. S. Gradshteyn and I. M. Ryzhik, Table of Integrals, Series, and Products, fourth edition (Academic Press, San Diego, 1980).

[27] J. C. Le Guillou and J. Zinn-Justin, Phys. Rev. Lett. 39, 95 (1977); Phys. Rev. B 21, 3976 (1980).

[28] J. M. Carmona, A. Pelissetto, and E. Vicari, Phys. Rev. B 61, 15136 (2000).

[29] The renormalized coupling constant $g$ can be expressed in terms of the second virial coefficient for a polydisperse ensemble of polymers, see Ref. [49].

[30] R. Guida and J. Zinn-Justin, J. Phys. A 31, 8103 (1998).

[31] A. Pelissetto and E. Vicari, Nucl. Phys. B 519, 626 (1998).

[32] A. Pelissetto and E. Vicari, Nucl. Phys. B 575, 579 (2000).

[33] M. Campostrini, A. Pelissetto, P. Rossi, and E. Vicari, Phys. Rev. E 57, 184 (1998). 
[34] M. Campostrini, A. Pelissetto, P. Rossi, and E. Vicari, Phys. Rev. B 54, 7301 (1996).

[35] This ratio is universal. However, it should be noted that we are comparing here the linear and the ring conformation for the same microscopic model, i.e. for polymers of the same chemical composition.

[36] S. Caracciolo, M. S. Causo, and A. Pelissetto, Phys. Rev. E 57, R1215 (1998), cond-mat/9703250.

[37] D. MacDonald, S. Joseph, D. L. Hunter, L. L. Moseley, N. Jan, and A. J. Guttmann, J. Phys. A 33, 5973 (2000).

[38] O. Jagodzinski, E. Eisenriegler, and K. Kremer, J. Phys. (France) I 2, 2243 (1992).

[39] J. L. Cardy and A. J. Guttmann, J. Phys. A 26, 2485 (1993).

[40] K. Y. Lin, Physica A 275, 197 (2000).

[41] B. Nienhuis, Phys. Rev. Lett. 49, 1062 (1982); J. Stat. Phys. 34, 731 (1984).

[42] M. Bishop and J. H. R. Clarke, J. Chem. Phys. 94, 3936 (1991).

[43] A. J. Guttmann and I. G. Enting, J. Phys. A21, L165 (1988).

[44] H. Saleur, J. Phys. A 20, 455 (1987).

[45] A. R. Conway and A. J. Guttmann, J. Phys. A 26, 1535 (1993).

[46] A. R. Conway and A. J. Guttmann, Phys. Rev. Lett. 77, 5284 (1996).

[47] I. Guim, H. W. J. Blöte, and T. W. Burkhardt, J. Phys. A 30, 413 (1997).

[48] S. Caracciolo, M. S. Causo, P. Grassberger, and A. Pelissetto, J. Phys. A 32, 2931 (1999).

[49] M. Muthukumar and B. G. Nickel, J. Chem. Phys. 80, 5839 (1984); 86, 460 (1987). 